\title{
Modèle ou faire-valoir ? La référence à Homère dans les commentaires de Virgile de Servius à La Cerda
}

\section{Christiane Deloince-Louette}

\author{
C OpenEdition \\ Journals \\ Édition électronique \\ URL : http://journals.openedition.org/rhetorique/882 \\ DOI : $10.4000 /$ rhetorique.882 \\ ISSN : 2270-6909 \\ Éditeur \\ UGA Éditions/Université Grenoble Alpes \\ Édition imprimée \\ ISBN : 978-2-37747-177-5 \\ Référence électronique \\ Christiane Deloince-Louette, « Modèle ou faire-valoir ? La référence à Homère dans les commentaires \\ de Virgile de Servius à La Cerda », Exercices de rhétorique [En ligne], 13 | 2019, mis en ligne le 06 \\ décembre 2019, consulté le 12 septembre 2020. URL : http://journals.openedition.org/rhetorique/882 \\ ; DOI : https://doi.org/10.4000/rhetorique.882
}

Ce document a été généré automatiquement le 12 septembre 2020.

\section{c) (i) (-)}

Les contenus de la revue Exercices de rhétorique sont mis à disposition selon les termes de la Licence Creative Commons Attribution - Pas d'Utilisation Commerciale - Partage dans les Mêmes Conditions 4.0 International. 


\title{
Modèle ou faire-valoir ? La référence à Homère dans les commentaires de Virgile de Servius à La Cerda
}

\author{
Christiane Deloince-Louette
}

Inclinez-vous, écrivains romains, et vous, Grecs, inclinez-vous !

Je ne sais quoi est né de plus grand que l'Iliade ${ }^{1}$.

1 En posant une claire hiérarchie entre le maitre et l'élève, le modèle et l'imitateur, l'Ancien et le Moderne, ces vers de Properce donnent le coup d'envoi à une longue série de comparaisons entre le poète de l'Énéide et celui de l'Iliade et de l'Odyssée. Pratiquée de manière systématique chez les premiers commentateurs de Virgile que sont Servius et Macrobe, la comparaison avec Homère aide à cerner l'art de Virgile. À la Renaissance, la vogue de Virgile est indéniable mais, alors même qu'il est fort peu lu, Homère continue à jouir du prestige de l'antériorité, du moins jusqu'au milieu du siècle.

Une étude systématique de la référence à Homère dans les commentaires de Virgile va en effet montrer, c'est mon propos, à quel point la comparaison avec le grand Ancien a contribué à orienter la lecture de Virgile et, plus particulièrement, à définir les caractéristiques d'un style, celui de l'Énéide, voire celui de l'épopée. La rupture profonde entre les deux auteurs se joue dans la deuxième moitié du XVI ${ }^{e}$ siècle et au début $\mathrm{du} \mathrm{XVII}^{\mathrm{e}}$ siècle, à une époque où la Contre-Réforme catholique se nourrit de nouvelles références à la Rome antique et à la langue latine. Cette rupture a des implications idéologiques dont je vais essayer de rendre compte.

\section{Homère : la source et le modèle}

3 Les premiers grands commentaires de Virgile, ceux de Servius et de Tiberius Claudius Donatus, apparaissent à la fin $\mathrm{du}_{\mathrm{Iv}}^{\mathrm{e}}$ siècle ou au début $d u \mathrm{v}^{\mathrm{e}}$ siècle de notre ère ${ }^{2}$. Si les Interpretationes virgilianae de Donat, essentiellement rhétoriques, ne s'intéressent pas à 
Homère, il n'en va pas de même chez Servius pour qui Homère est à la fois une source et un modèle. Une source, car la matière homérique nourrit l'Énéide : Énée est d'abord un héros de la guerre de Troie et c'est d'après Homère que Virgile évoque les héros et les dieux. Le choix même du contexte montre l'allégeance à Homère. Virgile parle secundum Homerum, selon Homère, il en est le brillant second ${ }^{3}$. Pour Servius, c'est un motif de louange. Pour Melanchthon aussi, qui publie en 1530 des scholies à l'Énéide: Virgile emprunte à Homère sa matière, il écrit ex Homeri [libris], en puisant dans les livres d'Homère ${ }^{4}$. C'est ainsi qu'il en acquiert le savoir : comme Homère, Virgile sera philosophe et son poème embrasse toutes les connaissances accessibles concernant la vie civile, la guerre, la conduite morale, la piété, etc. Le savoir prêté à Homère par toute l'Antiquité fait aussi la richesse de Virgile, c'est ce que signifie parler après et d'après Homère $^{5}$.

4 En cela, Homère n'est pas seulement une source, c'est aussi un modèle. Dans le prologue à son commentaire, Servius l'énonce clairement : «L'intention de Virgile est d'imiter Homère et de glorifier Auguste en tirant argument de ses ascendants ${ }^{6}$." L'imitation d'Homère est valorisée comme telle, indépendamment de tout jugement sur sa qualité. Son enjeu est double. Il concerne d'abord le genre du poème héroïque : imitant Homère, Virgile choisit le metrum heroicum et le stilus grandiloquus, le mètre héroïque et le style élevé7. Mais ce style élevé est lui-même directement repris d'Homère : Servius, Macrobe un peu plus tard, font l'éloge des emprunts de Virgile, des transpositions qu'il a su faire du grec en latin, des comparaisons, des épithètes. Lorsque Virgile traduit Homère mot à mot, il est loué; lorsqu'il traduit mal, c'est-à-dire se permet de sortir des chemins homériques, il est blâmé. Ainsi lorsqu'après la tempête du chant I, il montre son Énée en proie au doute alors qu'au chant VI de l'Odyssée, Homère peignait en Ulysse le héros noble et héroïque (heroica persona) :

On reproche ici à Virgile d'avoir improprement transposé les vers d'Homère : "Sentant se dérober ses genoux et son cœur, Ulysse alors gémit en son âme vaillante » [Od., V, 297-298]. Car « il sent un froid qui dénoue ses membres » est bien autre chose que « sentant se dérober ses genoux » : et " tendant ses deux mains vers les astres, il profère ces mots » est faible alors que « en son âme vaillante » a plus de grandeur, comme il sied à un personnage héroïque. De plus, qui lèverait de jour les mains vers les astres ou qui, tendant ses mains vers le ciel, ne ferait une autre prière plutôt que de dire " Ô trois et quatre fois heureux " ? Et Ulysse se parle à luimême, de peur que ses compagnons ne l'entendent et, plus craintifs, ne perdent courage, quand Énée au contraire pousse de grands cris ${ }^{8}$.

$\mathrm{Au}$ livre $\mathrm{V}$ de ses Saturnales, consacré au parallèle entre Homère et Virgile, Macrobe rend bien compte de ce type de lecture et de la position plus ou moins implicite qu'elle assume :

[Virgile] ne pouvait pas, sur certains points, ne pas se montrer inférieur au poète que, dans toute son œuvre poétique, il a pris pour modèle unique. En effet, de toute son ardeur, c'est sur Homère qu'il a les yeux fixés : il veut l'égaler, non seulement en grandeur, mais en simplicité, en puissance, en majesté calme dans le style. 41. C'est à lui qu'il emprunte la noble variété, la diversité de ses héros, l'intervention des divinités, son autorité dans les questions mythologiques, l'expression naturelle des sentiments, la recherche des traditions, l'ampleur des comparaisons, l'éclat d'un style entraînant, la brillante perfection du détail ${ }^{9}$.

Bref, sans Homère, Virgile ne serait rien.

Cette position des commentateurs me semble résumée par l'expression homericum est utilisée par Servius pour souligner les traductions judicieuses que Virgile donne d'Homère. Cette expression, que l'on trouve encore chez Melanchthon, dit tout à la fois 
l'allégeance de Virgile à Homère et l'admiration pour Virgile qui a si bien rendu l'indépassable Homère. Elle manifeste chez les commentateurs de Virgile la conscience aiguë d'une hiérarchie : ils commentent Virgile tout à la fois en référence à Homère et comme les commentateurs d'Homère ont commenté le poète grec. L'exemple de Melanchthon est éclairant à cet égard : la brièveté de ses scholies (quelques mots en marge du texte de Virgile) rend encore plus visible l'usage implicite de la référence. Chez Melanchthon en effet, homericus est un adjectif laudatif. La comparatio homerica souligne une réussite particulière dans la fidélité au modèle, comme le montre l'ample comparaison du chant $\mathrm{X}$ de l'Énéide (v. 726 et suivants), qui associe Mézence à un lion à jeun et dont Jacques Perret souligne encore qu'elle est empruntée au chant XVII de l' Iliade. Elle est qualifiée par le commentateur de comparatio emphatica et Homerica, " comparaison frappante, qui vient $\mathrm{d}^{\prime} \mathrm{Homère}^{10}$ ». Accolée à l'adjectif emphatica qui désigne la force de l'expression, la référence à Homère prend aussi un sens technique : la comparaison homérique est un type de comparaison aisément reconnaissable et appréciable par tous. Dans les expressions homericum est ou comparatio homerica, l'adjectif suffit à désigner l'effet de style.

6 Pour expliquer Virgile, Melanchthon se réfère aussi à deux termes récapitulatifs : elegantia et eruditio, la forme et le fond. Eruditio, c'est le savoir, la science, la philosophie, tout ce qui fait d'Homère, et de Virgile à sa suite, le détenteur d'un savoir complet. L' elegantia, c'est la forme, le style, le choix des mots et de leur agencement dans la phrase, dans le vers, etc. ${ }^{11}$. Le terme, assez problématique au xvI ${ }^{\mathrm{e}}$ siècle, on y reviendra, semble ici désigner l'ensemble du travail du style, quand eruditio rassemble tous les savoirs. Dans cette perspective, les deux termes transcrivent, on peut en faire l'hypothèse, les termes grecs qu'utilisait le pseudo-Plutarque dans son traité Sur Homère quand il se proposait d'étudier chez le poète grec à la fois la variété de la diction (lexis) et la connaissance des choses, la poluphônia et la polumatheia ${ }^{12}$.

7 On voit donc comment se met en place une terminologie stylistique: loin de se contenter de commenter Virgile par rapport à Homère, les commentateurs de Virgile adaptent les critères de lecture des commentateurs d'Homère au point que le nom même d'Homère devient un mot-outil du commentaire. Mais c'est un mot dangereux parce que, antonomase, il est lui-même figure. Il renvoie non à un critère objectif mais à un postulat implicite et subjectif : tout ce qui est homérique est digne d'admiration. Il peut donc changer de sens : ce sera la leçon de Scaliger.

\section{La révolution scaligérienne}

8 La lecture nouvelle que Scaliger va faire de Virgile à partir d'Homère, ou plutôt contre Homère, a cependant été préparée. Chez Macrobe déjà, même si le poète grec était ouvertement reconnu comme le modèle incontestable du poète latin, le parallèle entre Homère et Virgile a fait apparaître des distinctions stylistiques importantes. Quand il arrive à Virgile de surpasser Homère, c'est qu'il est plus expressif, qu'il a plus d'ornements, plus de couleurs, plus de détails. Virgile transcrit Homère de manière plus concentrée (Vergilium in transferendo densius excoluisse), il sait faire preuve d'un grand raffinement (magno cultu ${ }^{13}$ ). Lorsque les deux poètes décrivent une course de chars (Odyssée, XIII, 81 et Énéide, V, 144), les détails choisis par Homère ressortissent, semble$\mathrm{t}$-il, au simple travail du style (elegantia) quand Virgile a peint (pinxit) une description 
complète (descriptio plena ${ }^{14}$ ). Le cultus de Virgile renvoie à sa recherche du détail expressif, à sa varietas.

Largement diffusés au XVI ${ }^{e}$ siècle (trente-trois éditions), les jugements de Macrobe vont contribuer à inverser la hiérarchie initiale ${ }^{15}$. On ne peut arracher la première place à Homère, mais on peut amplifier la gloire de Virgile. Ainsi Peletier dans son Art poétique (1555) donne-t-il Virgile comme modèle absolu de «l'Cuuvre héroïque et immortel ». Et après avoir montré comment Virgile excelle dans les quatre styles, il revient à la comparaison avec Homère :

Voilà comment Virgile a distingué son éloquence en toutes façons, non sans une certaine prévoyance et présage: s'apprêtant de satisfaire à tant de sortes de Lecteurs à tant d'appétits et à tant de jugements. En quoi il a imité cette grande ouvrière et mère Nature, quand il a fait un accord de tant de sortes et de si divers tons. Vrai est que nous le trouvons redevable de la meilleure partie à Homère inventeur, et premier écriteur du genre Héroïque : qui fait que bien souvent nous détournons notre admiration de lui : quand nous voyons le lieu où il a pris son patron: et en référons l'honneur à son origine. Fors que telle fois, quand nous trouvons les choses qu'il a prises d'autrui, si bien enrichies, si bien polies, et accommodées, et d'un si grand jugement : et quand nous voyons ce qui est du sien, si entier, si net, et si bien agencé : nous ne pouvons penser, sinon que quand il n'eût point eu d'exemplaire : il n'eût laissé de faire aussi bien qu'il a fait ${ }^{16}$.

Peletier s'efforce visiblement ici de concilier deux positions antagonistes. Certes Homère est encore « l'inventeur et premier écriteur du genre Héroïque ", mais ce n'est plus qu'une concession. Car pour Peletier, Virgile n'a pas imité Homère, il a imité « cette grande ouvrière et mère Nature ». Dans ce face à face nouveau entre la Nature et le poète latin, l'« exemplaire » Homère s'évanouit discrètement, il n'est plus nécessaire.

Le coup de grâce que va porter Jules-César Scaliger au poète grec n'a en revanche rien de discret, il s'apparente plutôt à un enterrement en grande pompe. La comparaison d'Homère et de Virgile qui fait l'essentiel du livre V (Criticus) de La Poétique de 1560 s'inscrit dans la continuité du livre III (Idea) où Virgile est présenté au futur poète comme le seul auteur susceptible d'être imité ${ }^{17}$. Pour mieux donner la première place à Virgile et sous prétexte d'une comparaison, Scaliger se livre à une attaque en règle contre Homère dont les caractéristiques stylistiques permettent, par antithèse, de définir les beautés de Virgile.

11 Le chapitre 2 du livre $V$ pose la nouvelle hiérarchie :

Homère a beaucoup de génie, mais pour l'art il paraît l'avoir découvert plutôt que cultivé. Il ne faut donc pas s'étonner si l'on dit qu'on trouve en lui l'Idée de la nature, mais point d'art, cependant cette observation ne doit pas être prise pour une condamnation. Virgile avait reçu de lui un art grossier, il l'a élevé au plus haut point de perfection grâce aux études et au jugement d'une nature plus raffinée. Chose qui a été donnée à très peu, en lui retranchant beaucoup, il l'a rendu plus grand. Car la grandeur ne consiste pas dans la masse et l'abondance du discours, mais dans sa pureté et sa sobriété. Il a su aussi choisir ses idées de telle sorte que leur rayonnement donne à ses poèmes leur brillant éclat ${ }^{18}$.

Scaliger établit ici des distinctions que les siècles suivants vont entériner : l'opposition entre Homère et Virgile recoupe pour une part l'opposition entre la nature (ingenium, natura) et l'art (ars). Mais Scaliger est plus habile : loin de dénier tout art à Homère, il lui accorde un "art grossier» (artem rudem) dont l'examen va mettre en valeur le raffinement, l'éclat et la densité du poème virgilien.

Homère éparpille, Virgile concentre, l'un disperse, l'autre unifie. Homère a enseigné les deux formes de vie que nous pouvons embrasser, la morale civile dans 
l'Odyssée, la militaire dans l'Iliade, et il les a en quelque sorte incarnées en deux hommes; Virgile les a réunies en un seul personnage, en y joignant ainsi que je l'ai dit ailleurs la piété ${ }^{19}$.

Deux grandes oppositions stylistiques sont ainsi mises en place : aux longueurs et au relâchement d'Homère s'opposent la densité et la brièveté de Virgile, à la simplicité et au dépouillement homériques, la richesse et la majesté de Virgile. Au dixième livre de l' Iliade, dit Scaliger, «tout est nu; tout est languissant » ( nuda omnia ; omnino languet »). Les adjectifs nuda, rudia, infelicia (nus, grossiers, malheureux) condamnent la description du camp de Rhésus. En revanche, dans l'épisode comparable du chant IX de l'Énéide (l'expédition de Nisus et d'Euryale): "Chez notre poète, tout est divin: cadencé, varié, inattendu ${ }^{20} »$. Les vers d'Homère décrivant le bouclier d'Achille sont choses frivoles et puériles («frivola et puerilia»), les termes qu'il utilise sont ceux du peuple ("plebeiis verbis»). Mais lorsque Virgile peint le bouclier d'Énée, c'est un ouvrage céleste et les termes dont il use sont divins ("divinis verbis ${ }^{21}$ »). De même, à côté d'Andromaque qui pleure la mort de son époux comme une femme du peuple (« plebeiam mulierculam »), la mère d'Euryale fait entendre une plainte sublime (" conquestio sublimis $\left.{ }^{22} »\right)$. Face au vrai poète qu'est Virgile («verum poetam »), Homère n'est qu'un conteur de foire («foraneum narratorem»). Et si Scaliger reconnaît parfois à Homère une simplicité positive, il montre immédiatement comment Virgile a donné à la même matière plus de grandeur et de raffinement (grandior, cultior ${ }^{23}$ ).

Ces exemples, et il y en a beaucoup d'autres, prouvent que, loin d'être le poète qui excelle dans tous les styles comme le présentait Quintilien, Homère est pour Scaliger un représentant du style simple (humilis, en grec iskhnos). À propos d'une course de chars (Iliade XXIII, v. 500-501 et Énéide V, v. 144-147) Scaliger oppose ainsi la sécheresse (iskhnologia) d'Homère et l'animata oratio de Virgile, la phrase simple d'Homère et celle pleine de vie de Virgile. La conclusion est sans appel : «Les beautés des vers de Virgile et la plate sécheresse d'Homère ne sont pas comparables ${ }^{24}$. " À Homère le commun, le simple, le peu travaillé ; à Virgile l'ampleur, la force, la majesté, la grandeur. Il atteint seul à la noblesse du poème héroïque.

Mais Scaliger place aussi la polémique sur un autre terrain. À propos de la Rumeur de l' Énéide imitée de la Discorde de l'Iliade:

Quant aux niaiseries que disent les Grammairiens chez Aulu-Gelle sur l'Éris homérique et la Rumeur virgilienne, elles sont fort ridicules. Voici le passage sur la Discorde au IV de l'Iliade:

Elle, petite d'abord, se dresse; mais ensuite / elle a touché le ciel de la tête et elle marche toujours sur la terre.

Ils se récrient parce que Virgile a dit de la Rumeur qu'elle cache sa tête dans les nuées; pourtant Homère, qui est sa source, place la tête d'Éris au ciel. Je vous répondrai pour ma défense : je ne l'ai pas imité. Je ne veux pas l'imiter. Cela ne me plaît pas. Il est invraisemblable de placer la tête de la Discorde au ciel. C'est ridicule. C'est bouffon. C'est homérique. C'est grec. Ce n'est pas virgilien. Ce n'est pas romain. En outre ils sont aveugles à la force du jugement virgilien. S'il a caché la tête de la Rumeur dans les nuages, c'est parce que la tête de la Rumeur, c'est-à-dire l'origine des on-dit, est en général inconnue; et il a dit les nuées, et non le ciel, parce que depuis la terre des cris peuvent parvenir jusqu'aux nuages ${ }^{25}$.

Ce passage est intéressant à plusieurs égards: d'abord parce qu'on y retrouve l'expression homericum est, dont on a vu plus haut l'emploi glorieux qu'en faisaient Servius et Melanchthon. Or ici, homericum est devenu synonyme de ridiculum et de fatuum (bouffon). Mieux, il est remplacé par virgilianum, comme si, chez Scaliger, qui donne ses lettres de noblesse à l'adjectif, seul l'adjectif «virgilien » pouvait 
véritablement qualifier le style inimitable et inimité de Virgile! Deuxièmement, on remarque ici que la confrontation entre les deux poètes cède la place à une confrontation entre deux langues. Scaliger oppose non seulement Homère à Virgile, mais le grec au romain - et même le petit grec («graeculum») avec un évident mépris. En 1560, la Poétique de Scaliger transforme la rivalité entre deux poètes en une rivalité linguistique: la langue latine (ou romaine, puisque Scaliger ne considère la langue latine que dans l'état de perfection qu'elle a atteint à l'époque d'Auguste) l'emporte sur la langue grecque ${ }^{26}$.

À l'évidence, le parallèle entre Homère et Virgile chez Scaliger a fait d'Homère le fairevaloir, et même le repoussoir de Virgile, ce qui permet à l'Italien de définir le genre du poème héroïque sur des bases exclusivement latines. Et même si Ronsard, composant $L a$ Franciade quelques années plus tard, dit avoir "patronné [son] œuvre plutost sur la naïve facilité d'Homere, que sur la curieuse diligence de Virgile ", le poète futur imitera désormais non Homère mais Virgile, désigné par Scaliger comme "exemple, règle, principe et fin ${ }^{27}$ ». Les positions de Scaliger vont marquer pour longtemps la lecture de Virgile comme celle d'Homère.

\section{Homère et Virgile : la distinction de deux styles au début du XVII ${ }^{\mathrm{e}}$ siècle}

En 1583 paraît à Bâle le premier commentaire continu des poèmes homériques depuis celui d'Eustathe de Thessalonique au XII ${ }^{\mathrm{e}}$ siècle. C'est l'œuvre d'un jeune protestant français, Jean de Sponde ${ }^{28}$. De 1612 à 1617, le Père Juan Luis de La Cerda donne à son tour un commentaire complet et continu de l'Énéide qui, tout en s'appuyant sur la lecture de Scaliger, n'ignore pas le commentaire d'Homère par Sponde ${ }^{29}$. La confrontation de ces deux ouvrages va nous permettre de préciser les termes du divorce entre nos deux poètes.

\subsection{L'Homère de Sponde}

16 Dans ses « Prolégomènes à Homère ", Sponde fait incidemment référence à Scaliger qui, «s'il a bien parlé de la Poétique, a mal parlé d'Homère ${ }^{30}$ ». Le propos de Sponde est au contraire tout entier orienté vers la défense d'Homère et ses amples "Prolégomènes » qui font l'éloge de la Poétique (Poetica) puisent tous leurs exemples chez les auteurs grecs et tout particulièrement chez le premier d'entre eux. Il est clair qu'en rédigeant son commentaire, il a en tête les critiques de Scaliger. Sans faire de parallèle systématique avec Virgile mais en le citant très abondamment (c'est l'«Homère latin »!), Sponde tente en effet de réhabiliter Homère sur plusieurs plans : 1) celui de la langue : les poèmes homériques montrent la richesse de la langue et de la littérature grecques ; 2) celui de la piété : les héros d'Homère sont pieux et Homère lui-même était un homme pieux car en maints passages de son œuvre, il a manifesté une compréhension de la divinité presque chrétienne ; 3) celui du style : Sponde ne discute pas la lecture de Scaliger, mais il va justifier et louer la « simplicité » d'Homère.

Nous nous intéresserons ici aux deux derniers points, la question de la langue grecque risquant de nous entraîner fort loin de Virgile. Au reste, nous verrons que, dans l'esprit de Sponde, la question du style et celle de la piété sont liées. Dans l'ensemble de son 
commentaire en effet, Sponde souligne de manière récurrente l'elegantia d'Homère qui semble désigner pour lui la quintessence du style homérique. Chez Homère, les comparaisons sont elegantes et aptae (belles et appropriées), et sa description du bouclier d'Achille montre un artificium elegans (une belle composition). Qu'est-ce à dire ? Il faut revenir au sens premier du terme dans l'Antiquité.

Pour la Rhétorique à Herennius (IV, 7), l'elegantia tient à deux éléments: la latinité (latinitas), donc la correction de la langue, et la clarté (explanatio). Au livre III du De l'Orateur, évoquant l'agencement des mots dans la phrase, le Crassus de Cicéron n'utilise pas le terme mais en donne pratiquement une définition:

De même, dans toutes les parties d'une phrase, ce qui est utile et presque indispensable ne va pas sans une sorte de charme et d'agrément ${ }^{31}$.

L'elegantia, c'est le charme qui naît du juste choix des mots et de leur arrangement dans la phrase. C'est le degré zéro du style, mais aussi sa condition nécessaire, la base sans laquelle il n'y a pas d'ornement possible. Dans L'Orateur, Cicéron l'associe au refus de la parure voyante, de l'ornement trop visible : l'elegantia, c'est l'effet de style qui ne se déclare pas comme tel.

Les humanistes vont s'emparer de la notion: si Lorenzo Valla l'associait surtout à l'expression d'une pure latinité, Scaliger la définit comme l'agencement non commun de termes choisis («verborum electorum non vulgaris coaptatio ${ }^{32}$ )». Le sens est proche de celui qu'on trouve dans L'Orateur de Cicéron: le travail du style ne concerne pas seulement le choix des mots, mais aussi leur agencement dans la phrase. Il est lié au style bas ou simple, à la subtilis oratio ${ }^{33}$.

Or le terme, principalement sous sa forme adjectivale ou adverbiale (elegans, eleganter) est l'outil le plus fréquemment utilisé par Sponde pour qualifier le style d'Homère. Le commentaire au v. 5 du chant $\mathrm{X}$ de l''liade glose ainsi la comparaison que fait Homère entre les soupirs d'un Agamemnon angoissé par la situation et les éclairs que lance Jupiter :

À quel point tous ces éléments concourent à la beauté de l'expression, le lecteur cultivé peut aisément en juger et même, d'après ce seul passage, s'assurer du génie $\mathrm{d}^{\prime}$ Homère $^{34}$.

Et trois vers plus loin, commentant l'image de « la gueule de la guerre »:

La gueule de la guerre [...] est une belle expression que le Poète a inventée pour rendre ce qu'on pourrait appeler sa voracité. [...] D'une façon similaire, dans son discours pour le poète Archias [au $\$ 21$ ], Cicéron parle aussi de la gueule et du gosier de la guerre. "C'est à la louange du peuple romain, dit-il, que la ville des Cyzicéniens, nos si grands alliés, a été préservée de tout assaut royal par le conseil du même homme, et arrachée à la gueule et au gosier de la guerre ». Par conséquent tu vois bien qu'on peut dire à bon droit qu'Homère est le père des belles expressions et qu'il a emprunté longtemps avant les autres la voie du discours harmonieux ${ }^{35}$.

L'harmonie (concinnitas) découle donc de l'elegantia : il n'y a pas d'harmonie sans choix et disposition judicieux des mots. Et dans toute l'lliade, déclare Sponde dans son commentaire au début du chant IX (les ambassades à Achille), il n'y a pas de chant (rhapsodia) "où l'expression soit plus belle et dans lequel éclate davantage l'art Rhétorique $^{36}$ ». L'elegantia en vient à désigner l'ensemble du travail du style - la mise en œuvre de l'art rhétorique - que le chant IX révèle avec éclat ${ }^{37}$.

Or cette beauté de l'expression homérique, cette habileté stylistique qui conjoint l'harmonie et la clarté, Sponde l'associe à la simplicité d'un Poète qui, avant tout, transmet un savoir: "Poeta docet, Poeta docet simpliciter ${ }^{38}$ ». Pour Sponde comme pour l'ensemble de l'Antiquité, Homère est un maître et les poèmes homériques relèvent 
d'abord du docere. Sponde ne récuse pas Scaliger : il reconnaît la simplicité homérique mais tourne à l'avantage du poète grec ce qui était pour Scaliger un motif de condamnation.

Il est temps alors de revenir à la piété. On se souvient que là résidait pour Scaliger un des apports principaux de Virgile : l'Énéide ne se contentait pas d'associer en un même poème une Iliade et une Odyssée, elle mettait en scène la piété par l'intermédiaire de son héros. Sponde rétorque en montrant sans cesse les héros d'Homère respectueux du culte et des dieux. Pour le protestant, la beauté simple du style d'Homère va de pair avec la simplicité de héros qui vivent sans luxe superflu, comme au temps de Moïse ou des premiers chrétiens. Au reste, à plusieurs reprises, Sponde opposera au luxe des princes de son époque la simplicité et l'absence d'ornements superflus chez les Grecs d'Homère ${ }^{39}$.

La lecture de Sponde donne donc une nouvelle cohérence à Homère : le fond justifie la forme, la vie simple et pieuse des héros homériques ainsi que la dimension didactique et morale de sa poésie justifient le recours au style simple. Cette réhabilitation d'Homère n'aura cependant pas grand succès. Au début du XVII siècle, elle sert à son tour de repoussoir au jésuite espagnol Juan Luis de La Cerda lorsqu'il publie entre 1612 et 1617 son ample commentaire de l'Énéide ${ }^{40}$.

\subsection{Le Virgile de La Cerda}

Professeur de rhétorique, de poésie et de littérature grecque, La Cerda avait déjà en 1608 publié une édition commentée des Bucoliques et des Géorgiques. L'édition de l'Énéide achève donc une édition des œuvres complètes de Virgile dont l'unité est assurée par l'« Éloge de Virgile » qui ouvre le premier volume. Virgile y est comparé, sans surprise, à ces deux autres auteurs "récapitulatifs » que sont Cicéron et Homère. Sur ce dernier point, la source essentielle, et avouée, est Scaliger et on ne s'étonnera pas de trouver sous la plume du jésuite une condamnation en règle des absurdités d'Homère. Dans son commentaire à l'Énéide, les références à Homère (au nombre d'une cinquantaine si l'on ajoute celles qui concernent les héros homériques) renvoient presque exclusivement au poète grec comme source et non comme modèle. Et même dans ce cas, la critique est là, opposant par exemple à Énée un Ulysse rusé et menteur. Dans son éloge initial de Virgile, le jésuite ajoute même que, si beaucoup d'autorités de l'Église ont fait grand cas de Virgile, tout particulièrement Tertullien, c'est que Virgile, à la différence d'Homère, a su mettre en scène un pieux héros (Énée est " piissimorum piissimus », " le pieux parmi les pieux ») et faire de l'Énéide une œuvre pieuse ${ }^{41}$. Sur le plan stylistique, La Cerda dépasse même Scaliger dans l'énumération des caractéristiques négatives du style homérique :

Ses propos sont nus, communs, faciles, tout simples, ébauchés seulement et non parfaits, faibles, chétifs, légers et niais au dernier degré, secs, misérables, bouffons, menteurs, puérils, languissants, dépourvus d'art et de sentiment; ce sont des contes de bonne femme, de petits mots desséchés, grossiers, des pensées décharnées, un éclat sans recherche, des choses futiles et bavardes, un style misérable et qui sent la taverne, une façon de parler contrainte et sans douceur, des figures barbares. Ses répétitions sont absurdes et montrent sa folie. Il est plein des funestes balivernes des Grecs ${ }^{42}$. reprises la beauté (elegantia) de ses images ou de ses comparaisons, son attention se 
porte tout particulièrement sur deux qualités, pour lui proprement virgiliennes, l' aptum (ou convenance, prépon en grec) et l'expression des émotions, deux qualités, on ne s'en étonnera pas, qu'il dénie à Homère. L'aptum se lit dans les comparaisons, des comparaisons qui, bien souvent - mais cela n'est pas dit - pourraient être des comparaisons homériques. Ainsi dans le commentaire au v. 565 du chant X où Énée est comparé à Égéon, monstre aux cent bras, La Cerda loue une « comparaison tout à fait propre à expliciter la fureur d'Énée" (aptissima comparatio ad Aeneae furorem explicandum) mais explique que Virgile ne compare jamais Énée à un lion, un tigre ou d'autres bêtes cruelles et farouches car ce type de comparaison n'est pas approprié à la dignité du prince Énée qui représente l'empereur Auguste. Il est donc réservé aux «méchants » comme Turnus ou Mézence ${ }^{43}$. Homère, dont tous les héros sont comparés au moins une fois à un lion, est directement visé.

Mais ce qui intéresse par-dessus tout La Cerda, c'est la mise en valeur de la qualité dominante du style de Virgile, l'expression de l'émotion. Quand Homère, sec et sans âme, écrit sans aucune recherche ("sine ullo apparatu»), Virgile excelle "in movendis affectibus ", dans l'art de faire naître l'émotion ${ }^{44}$. Dans l'ensemble du commentaire, on trouve 256 occurrences du terme affectus (contre 18 pour elegantia). Ainsi dans l'explication de la fin du discours d'Énée à Vénus, au v. 388 du chant I, alors que le héros énumère les épreuves qu'il a subies :

Dans cette ultime partie du discours, il laisse libre cours à son éloquence et suscite les plus vives émotions; tels sont les moyens qui peuvent provoquer les larmes ${ }^{45}$.

Si la beauté du style et la simplicité d'Homère le plaçaient du côté du docere, le pathétique et le raffinement de Virgile le poussent à l'évidence du côté du movere.

On voit l'implication idéologique de la confrontation de ces lectures : l'esthétique belle et simple s'apparente à celle de la Réforme, désireuse d'enseigner à tous un Évangile dont la simplicité elle-même n'est plus à prouver. En revanche, l'esthétique de la grandeur et de l'émotion, liée à la glorification de la langue latine, est bien celle de la Contre-Réforme. À la même époque, les travaux d'Isaac Casaubon sur Polybe (1609) révèlent des préoccupations identiques : Polybe est du côté de l'elegantia, son éloquence est " âpre et toute militaire, elle sent les champs de batailles et refuse le recours aux fards et au fer à friser »; Tite-Live en revanche est « divinement éloquent », il est du côté du grand style ${ }^{46}$.

De Servius à La Cerda, du ve au XvII e siècle, la lecture que l'on a fait de l'auteur de l' Énéide a considérablement changé. Le brillant second d'Homère a vu sa gloire s'accroître au fur et à mesure que les lecteurs d'Homère se raréfiaient. Lorsque les humanistes de la Renaissance redécouvrent la langue et la littérature grecques, l'admiration pour le premier des poètes cède vite la place à la conscience de son « insuffisance ». C'est cependant la confrontation avec Homère qui permet, a contrario, de nommer les différences et de définir la qualité principale de l'art virgilien, l'art de faire naître l'émotion, que les Jésuites de la Contre-Réforme vont exploiter à leur profit. Dans cette époque troublée qui suit immédiatement les guerres de religion, il semble que le conflit entre protestants et catholiques se soit déplacé non seulement sur le terrain de la parole - c'est la grande époque de la controverse religieuse - mais aussi sur celui, pourtant a priori moins polémique, du commentaire. 


\section{BIBLIOGRAPHIE}

Cicéron, De l'Orateur, Paris, Les Belles Lettres (C. U. F.), 1950-1956.

Cicéron, L'Orateur, Paris, Les Belles Lettres (C. U. F.), 1964.

La Cerda (Juan Luis de), Virgilii (Publii) Maronis Bucolica et Georgica, Argumentis, Explicationibus, Notis illustrata. Auctore Ludovico de la Cerda [...], Lyon, H. Cardon, 1619 (t. I première édition en 1608) ; Priores sex libri Aneidos argumentis explicationibus notis illustrati auctore Joanne Ludovici de La Cerda, Lyon, H. Cardon, 1612 (t. II) ; Posteriores sex libri Aneidos argumentis explicationibus notis illustrati auctore Joanne Ludovici de La Cerda, Lyon, H. Cardon, 1617 (t. III).

Marmontel, Éléments de littérature française (1787), édition présentée et établie par S. Le Ménahèze, Paris, Éditions Desjonquères, 2005.

Melanchthon, Philippi Melanthonis Opera quae supersunt omnia, vol. XIX, éd. Karl Gottlieb Bretschneider puis Heinrich Ernst Bindseil, Brunswick, C. A. Schwetschke et fils (Corpus Reformatorum), 1853.

Scaliger (Julius Caesar), Poetices libri septem, éd. en six volumes de Luc Deitz et Gregor Vogt-Spira, Stuttgart-Bad Cannstatt, Frommann-Holzboog, 1994-2011 ; La Poétique, V, Le Critique, traduction française du livre V par J. Chomarat, Genève, Droz, 1994.

Servius, Servii grammatici qui feruntur in Vergilii carmina commentarii, 4 volumes, éd. Thilo-Hagen, Leipzig, Teubner, 1923.

Sponde (Jean de), Homeri quae extant omnia, [...] perpetuis item iustisque in Iliada simul et Odysseam Io. Spondani Mauleonensis commentariis [...], Bâle, Eusebius Episcopus, 1583 ; traduction française, Commentaires aux poèmes homériques, par $\mathrm{Ch}$. Deloince-Louette avec la collaboration de M. Furno, Paris, Classiques Garnier, 2018 (3 vol.).

Traités de poétique et de rhétorique de la Renaissance, introduction, notices et notes de Fr. Goyet, Paris, Librairie Générale Française, 1990.

\section{Critique moderne}

Balavoine (Claudie) et Laurens (Pierre), éd., La Statue et l'empreinte : la Poétique de Scaliger, Paris, Vrin, 1986

Ford (Philip), De Troie à Ithaque. Réception des épopées homériques à la Renaissance, Genève, Droz, 2007.

Lecointe (Jean), L’Idéal et la différence, Genève, Droz, 1993.

Lecompte (Stéphanie), La Chaîne d'or des poètes. Présence de Macrobe dans l'Europe humaniste, Genève, Droz, 2009.

Parenty (Hélène), Isaac Casaubon helléniste. Des studia humanitatis à la philologie, Genève, Droz, 2009.

Saladin (Jean-Christophe), La Bataille du grec à la Renaissance, Paris, Les Belles Lettres, 2000. 


\section{NOTES}

1. Properce, Élégies, II, 34, v. 65-66: «Cedite Romani scriptores, cedite Grai!/nescio quid maius nascitur Iliade ». Nous traduisons. Ces vers sont encore cités par le P. Rapin dans la conclusion de sa Comparaison des poëmes d'Homere et de Virgile, Paris, Claude Barbin, 1664.

2. Servii grammatici qui feruntur in Vergilii carmina commentarii, 4 volumes, éd. Thilo-Hagen, Leipzig, Teubner, 1923 ; Ti. Cl. Donatus, Interpretationes virgilianae, éd. H. Georgi, Leipzig, Teubner, 1905.

3. Les occurrences de la formule sont innombrables : ainsi dans le commentaire au v. 4 du livre I : «VI SUPERUM uiolentia deorum, secundum Homerum, qui dicit a Iunone rogatos esse deos in odium Troianorum " (Servius, op. cit., vol. I, p. 10); au v. 30 du livre I encore : « ATQUE INMITIS ACHILLI bene secundum Homerum segregat duces a populo » (op. cit., vol. I, p. 24) ; au v. 108 du livre II : « SAEPE FUGAM DANAI hoc secundum Homerum verum est, apud quem suadet Agamemnon Graecis relicto bello fugere » (op. cit., vol. I, p. 236), etc.

4. Philippi Melanthonis Opera quae supersunt omnia, vol. XIX, éd. K. G. Bretschneider puis H. E. Bindseil, Brunswick, C. A. Schwetschke et fils (Corpus Reformatorum), 1853, col. 435-472 (première édition 1530, Haganoae per Ioh. Secerium) : voir par exemple les notes aux v. 626 et 894 du livre VI, au v. 264 du livre X, aux v. 170, 206, 347, 725, 731 du livre XII.

5. C'est la tradition du poète "récapitulatif " (le terme est de J. Lecointe, L'Idéal et la différence, Genève, Droz, 1993, p. 162-172) telle que la transmet par exemple Quintilien, Institution oratoire, X, 1,46 .

6. Servius, op. cit., vol. I, p. $4:$ : [...] intentio Vergilii haec est, Homerum imitari et Augustum laudare a parentibus. »

7. Ibid.

8. Servius, commentaire au chant I, v. 92 (op.cit., vol. I, p. 47) : «Reprehenditur sane hoc loco

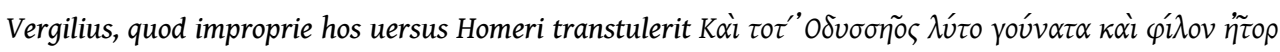

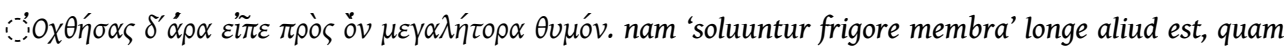

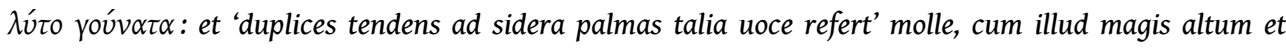

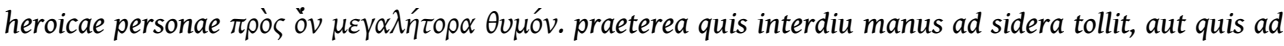
caelum manum tendens non aliud precatur potius, quam dicit 'o terque quaterque beati'? et ille intra se, ne exaudiant socii et timidiores despondeant animo, hic uero uociferatur. »

9. Macrobe, Saturnales, V, XIII, 40-41, édition et traduction de H. Bornecque, Paris, Garnier, 1937 : «Et re vera non poterat non in aliquibus minor videri, qui per omnem poesim suam hoc uno est praecipue usus archetypo. Acriter enim in Homerum oculos intendi, ut aemularetur ejus non modo magnitudinem, sed et simplicitatem et praesentiam orationis et tacitam majestatem. 41. Hinc diversarum inter heroas suos personarum varia magnificatio, hinc deorum interpositio, hinc auctoritas fabulosorum, hinc affectuum naturalis expressio, hinc monumentorum persecutio, hinc parabolarum exaggeratio, hinc torrentis orationis sonitus, hinc rerum singularum cum splendore fastigium. "

10. Voici quelques exemples supplémentaires de l'usage de l'adjectif homericum chez Melanchthon (op. cit.) : «Homericum est, qui fingit Ulyssem circumdari nebula a Minerva " (scholie à I, 411, «Imitation d'une fiction homérique: Minerve enveloppe Ulysse d'une nuée ») ; « Homericum est; finguntur enim Dii venti quidam illi » (scholie à IV, 278, «Imitation d'une fiction homérique : certains vents sont des Dieux»); en IX, 106 à propos du vers " adnuit et totum nutu tremefecit Olympum» («il fit un signe et le mouvement de sa tête fit trembler l'olympe tout entier»), il note simplement «Homericum»; en II, 496 : «Comparatio Homerica»; en IV, 441 : « Homerica comparatio ».

11. Les deux termes apparaissent ensemble au tout début du commentaire au livre IV de l'Énéide (ibid., col. 446) : "In hoc libro plus est elegantiae quam eruditionis ; in V. et VI. plus eruditionis ».

12. Pseudo-Plutarque, Essay on the Life and Poetry of Homer, éd. de J. J. Keany et R. Lamberton, Atlanta (GA), Scholars Press, 1996, p. 70.

13. Saturnales, V, XI, 1-9, op. cit., p. 101-105. 
14. Ibid., V, XI, 21.

15. Voir S. Lecompte, La Chaîne d'or des poètes. Présence de Macrobe dans l'Europe humaniste, Genève, Droz, 2009.

16. Jacques Peletier du Mans, Art poétique, dans Traités de poétique et de rhétorique de la Renaissance, introduction, notices et notes de Francis Goyet, Paris, Librairie Générale Française, 1990, p. 313.

17. Julius Caesar Scaliger, Poetices libri septem, éd. en six volumes de L. Deitz et G. Vogt-Spira, Stuttgart-Bad Cannstatt, Frommann-Holzboog, 1994-2011.

18. Ibid., V, 2, t. IV, p. 46 : "Homeri ingenium maximum, ars eiusmodi, ut eam potius invenisse quam excoluisse videatur. Quare neque mirandum est, si in eo naturae idea quaedam, non ars exstare dicatur. Neque censura haec pro calumnia accipienda. Vergilius vero artem ab eo rudem acceptam lectioris naturae studiis atque iudicio ad summum extulit fastigium perfectionis. Quodque perpaucis datum est, multa detrahendo fecit auctiorem. Neque enim in mole frequentiave orationis, sed in castitate atque frugalitate magnitudo constituta est. Rerum quoque delectum eiusmodi habuit, e quarum splendore luculentus ille nitor suis poematibus adiungeretur. » Pour toutes les citations de Scaliger, la traduction est celle qu'a proposée Jacques Chomarat, dans J.-C. Scaliger, La Poétique, V, Le Critique, Genève, Droz, 1994 (ici, p. 12).

19. Scaliger, La Poétique, livre V, op. cit., p. 12 ; Poetices, op.cit., t. IV, p. 48 : « Fudit Homerus, hic collegit; ille sparsit, hic composuit. Homerus ergo cum vitae nostrae duas instituerit rationes, civilem prudentiam in Ulyssea, militarem in Iliade, easque tamquam duas species in duobus viris ostendisset, in uno utramque Aenea composuit Maro, cui etiam sicut alibi diximus addiderit pietatem. "

20. Voir ibid., t. IV, p. 158 : « Nostra divina omnia, numerosa, varia, inexspectata. »

21. Ibid., p. 194 et 196.

22. Ibid., p. 206. Et Scaliger ajoute (ibid., p. 208, trad. Chomarat, op. cit. p. 100) : « Omnia signate ex natura, ex arte, ex eruditione: sententiae, numeri, figurae, simplicitas, candor, ornatus incomparabilia atque uno ut absolvam Vergilii », " Tout est expressif, selon la nature, selon l'art, selon l'érudition ; les pensées, les rythmes, les figures, la simplicité, la limpidité, les ornements y sont incomparables; et pour tout dire d'un mot : virgilien. »

23. Ibid., p. 208-210 : la grandeur et le raffinement viennent aussi du rythme («numerus »).

24. Voir ibid., p. 212 : " [...] cum virtutibus horum carminum non est conferenda ieiuna illa humilitas. »

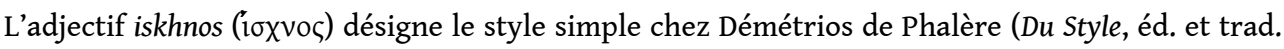
de P. Chiron, Paris, Les Belles Lettres, 2002), essentiellement par opposition au grand style.

25. Voir ibid., p. 152-154 : "Quae vero apud Gellium nugantur grammatici de Eride Homerica et Fama Vergiliana, valde ridicula sunt. De Contentione ille in quarto Iliadis: "

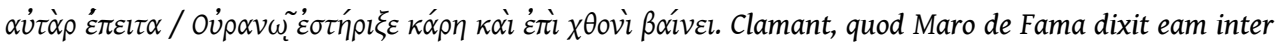
nubila caput condere, cum tamen Homerus unde ipse accepit in caelo caput Eridis constituat. Iam tibi pro me respondeo: Non sum imitatus. Nolo imitari. Non placet. Non est verum Contentionem ponere caput in caelo. Ridiculum est. Fatuum est. Homericum est. Graeculum est. Vergilianum non est. Romanum non est. Praeterea latet eos vis iudicii Maroniani. Abscondit enim Famae caput nubibus, propterea quod famae caput, id est auctor rumorum, fere incertus est. Et dixit nubila, non caelum, quia ad nubes usque pervenire a terra clamor potest. "

26. Sur cette question, qui dépasse le cadre du présent article, voir La Statue et l'empreinte: la Poétique de Scaliger, études réunies et présentées par C. Balavoine et P. Laurens, Paris, Vrin, 1986 ; J.-C. Saladin, La Bataille du grec à la Renaissance, Paris, Les Belles Lettres, 2000 ; Ph. Ford, De Troie à Ithaque. Réception des épopées homériques à la Renaissance, Genève, Droz, 2007, chapitre VI.

27. Scaliger, Poetices, V, 3, op. cit., t. IV, p. 300 ; Ronsard, préface à La Franciade, in CEuvres complètes, Paris, Gallimard, Bibliothèque de la Pléiade, t. I, p. 1183. Pour Ronsard, l'art est cependant toujours du côté de Virgile («curieuse diligence») et la nature du côté d'Homère ("naïve facilité »). Notons tout de même la parution en 1575 chez Christophe Plantin à Anvers d'une édition annotée de Virgile due à un ami de Ronsard, Vaillant de la Guerle (Valens Guellius), abbé 
de Pimpont, dont les annotations à l'Énéide consistent presque exclusivement à relever les emprunts à Homère.

28. Jean de Sponde, Homeri quae extant omnia,[...] perpetuis item iustisque in Iliada simul et Odysseam Io. Spondani Mauleonensis commentariis [...], Basileae, Eusebii Episcopii opera ac impensa, 1583. Pour une édition critique avec traduction française, voir Jean de Sponde, Commentaire aux poèmes homériques, éd. Ch. Deloince-Louette avec la collaboration de M. Furno, Paris, Classiques Garnier, 2018 (3 vol.).

29. P. Virgilii Maronis Priores sex libri Aeneidos Argumentis, explicationibus, notis illustrati, Auctore Ioanne Ludouico de la Cerda [...], LVGDVNI, sumptibus Horatii Cardon, 1612; P. Virgilii Maronis Posteriores sex libri Aeneidos Argumentis, explicationibus, notis illustrati, Auctore Ioanne Ludouico de la Cerda [...], LVGDVNI, sumptibus Horatii Cardon, 1617. Le premier volume est republié en 1619 chez le même éditeur. La traduction française est la nôtre.

30. Sponde, op. cit., " Prolégomènes à Homère ", p. 29.

31. Cicéron, De l'Orateur, III, 181, édition et traduction de H. Bornecque, Paris, Les Belles Lettres, 1956: «Hoc in omnibus item partibus orationis euenit, ut utilitatem ac prope necessitatem suauitas quaedam et lepos consequatur. »

32. Poetices, IV, 1, op.cit., p. 292. Sur les Elegantiae de Valla et celles de Dathi, voir J. Lecointe, op. cit., p. 594-604.

33. L'Orateur, 78-79, édition et traduction de A. Yon, Paris, Les Belles Lettres, 1964, p. 28. Du fait de cette simplicité même, la traduction d'elegantia reste difficile. J'ai opté pour une traduction neutre comme «beauté de l'expression » ou «belle expression » pour tenter de rendre ce degré zéro mais nécessaire du travail stylistique, assez proche cependant de notre moderne «bonheur d'expression ".

34. Sponde, op.cit., p. 175 : "Quae omnia quam eleganter conueniant, facile potest lector eruditus iudicare, et uel ex hoc solo loco ingenium Homericum explorare. "

35. Ibid., commentaire au v. 8 du chant X : «Os bello, [...] eleganter Poeta affingit, propter eius quasi uoracitatem. [...] Simili ratione Tullius quoque belli os et fauces appellat pro Arch. Poeta. Populi, inquit, Romani laus est, urbem amicissimam Cyzicenorum, eiusdem consilio ex omni impetu regio, ac totius belli ore et faucibus ereptam esse atque seruatam. Vnde uides elegantiarum parentem merito Homerum nostrum dici posse, et aliis hanc concinne loquendi uiam longo ante tempore praeiuisse. » Il y a sans doute un peu d'ironie chez Sponde à commenter Homère avec l'aide de Cicéron. Est-ce une pique contre Scaliger?

36. Ibid., p. 150 : "In toto hoc Iliadis opere nullam elegantiorem extare, et in qua maius artificium Rhetoricum eluceat. "

37. C'est aussi le sens que donnera au terme Marmontel dans ses Éléments de littérature française (1787) (édition présentée et établie par S. Le Ménahèze, Paris, Éditions Desjonquères, 2005, p. 441 sq.) : « ÉLÉGANCE - Celle du style suppose la correction, la justesse, la pureté de la diction, c'est-àdire la fidélité la plus sévère aux règles de la langue, au sens de la pensée, aux lois de l'usage et du goût ; mais tout cela contribue à l'élégance et n'y suffit pas. Elle exige encore une liberté noble, un air facile et naturel, qui, sans nuire à la correction, déguise l'étude et la gêne. »

38. Ces expressions se trouvent partout dans le commentaire de Sponde: voir Ch. DeloinceLouette, Sponde commentateur d'Homère, Paris, Champion, 2001, p. 207-210.

39. Voir par exemple ce commentaire au chant III de l'Iliade, v. 269 (ibid., p. 56) : « [...] tanta fuit simplicitas elegantis illius seculi», "[...] tant était grande la simplicité de cette époque sans affèterie ».

40. Pour la référence précise, voire supra note 28.

41. « P. Virgilii Maronis Elogia» (non paginé), chapitre II, op. cit., t. I. La Cerda éditera en 1624 les œuvres complètes de Tertullien.

42. Ibid., chapitre IV, «De Homero", op. cit., t. II : «Proponit nuda, plebeia, quovis ingenio parabilia, simplicia, incoata tantum et non perfecta, mollia, tenuissima, levissima, ineptissima, ieiuna, pessima, fatua, 
falsa, puerilia, languida, sine arte, sine affectu, nugas anicularum, verbula sicca, vasta, sententias aridissimas, apparatum sine apparatu, futilia, loquacia, orationem miseram et tabernariam, dictionem coactam et insuavem, figuras barbaras. Ineptit repetendo, insanitque. Plenus est fatali Graecorum nugacitate.»

43. Ibid., t. III, p. 495.

44. La Cerda, « P. Virgilii Maronis Elogia » (non paginé), respectivement chapitres IV et III (op. cit., t. I).

45. Ibid., t. II, p. 74 : « Est haec extrema pars orationis, ubi fontes aperit eloquentiae, maioresque affectus mouet ; et talia haec sunt, ut a quouis lacrymas possint excitare. »

46. Voir H. Parenty, Isaac Casaubon helléniste. Des studia humanitatis à la philologie, Genève, Droz, 2009, p. 189-191.

\section{AUTEUR}

\section{CHRISTIANE DELOINCE-LOUETTE}

Université Grenoble Alpes - UMR 5316 Litt\&Arts 